- Case Report

\title{
Synchronous Occurrence of Bilateral Malignant Otitis Externa: Report of a Rare Case
}

\author{
Nur Adillah Lamry', Khairunnisak Misron², Tengku Mohamed Izam Tengku Kamalden², Azliana Aziz', Rosdan Salim, ${ }^{1 * *}$ \\ 'Department of Otorhinolaryngology-Head and Neck Surgery, School of Medical Sciences, Universiti Sains Malaysia Health Campus, Kota Bharu, \\ Malaysia \\ ${ }^{2}$ Department of Otorhinolaryngology Head and Neck Surgery, Hospital Sultan Ismail, Johor Bahru, Malaysia
}

Malignant otitis externa (MOE) is a rare and potentially life-threatening disease of the ear and temporal bone. Bilateral simultaneous MOE is extremely rare. Due to bilaterally symmetrical facial nerve palsy, it can easily be missed at the initial presentation, causing delay in management. Here, we report a case of bilateral MOE managed aggressively with regular ear toileting, ear packing with a ribbon gauze soaked with topical antimicrobials, and long-term intravenous and oral antibiotics. The patient showed good improvement in pain control, facial nerve status, and ear findings.

Keywords: Otitis Externa; Ear; Facial Paralysis; Temporal Bone 


\section{INTRODUCTION}

Malignant otitis externa (MOE) usually affects immunocompromised individuals, typically the elderly with diabetes mellitus. ${ }^{1)}$ It is predominantly a unilateral disease, and in rare cases, presents with bilateral simultaneous facial nerve palsy, in which it can easily be missed. MOE should be treated aggressively with systemic antibiotics and local disease control in the ear canal using regular ear toileting and topical application of antibiotics. Failure to perform treatment can cause progression to osteomyelitis of the skull base with serious consequences. After commencing the treatment, our patient was monitored closely for resolution of the chief symptoms, which were ear pain and discharge and facial asymmetry, and improvements in blood parameters.

\section{CASE REPORT}

A 60-year-old woman presented to the Otorhinolaryngology Clinic with pain in the left ear, which was followed shortly with bilateral ear discharge for the past 3 months and reduced hearing bilaterally. A general practitioner had diagnosed her with bilateral chronic suppurative otitis media. She had been administered with antimicrobial ear drops and oral antibiotics for 2 weeks with no signs of improvement. She showed difficulty moving the facial muscles bilaterally, particularly in the lower portion of the face. Facial nerve palsy was missed at the initial presentation, probably because of bilateral facial muscle involvement, leading to a symmetrical appearance of the face. There
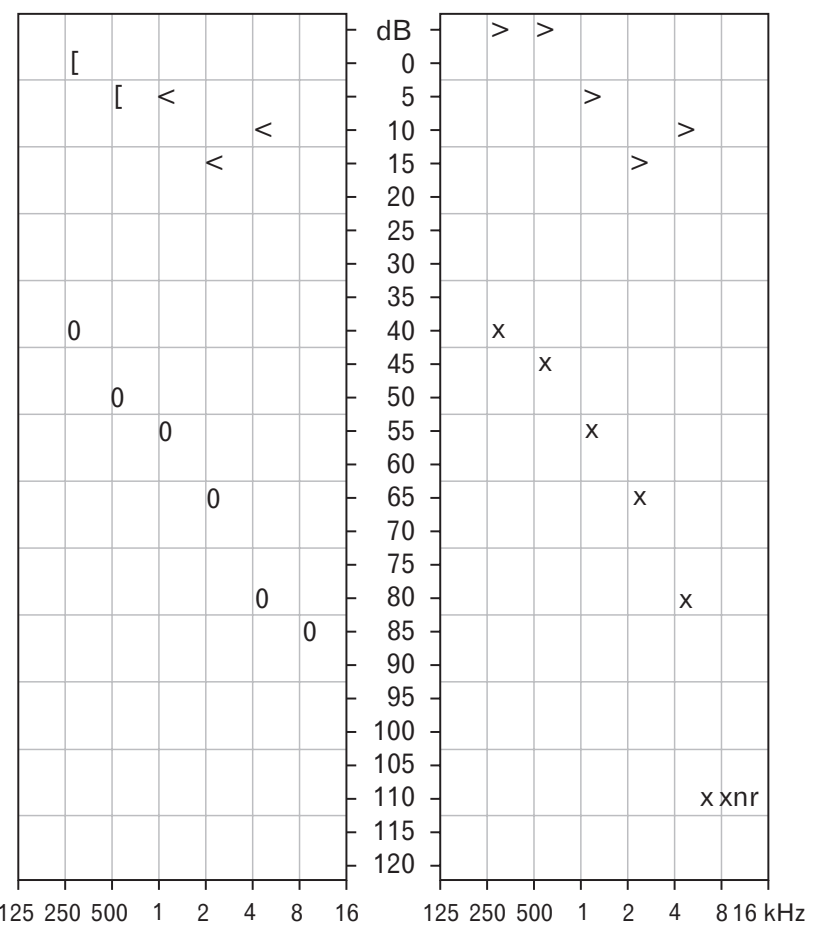

Figure 1. Pure-tone audiometry of both right and left ears show mild to severe conductive hearing loss, as evident by the air-bone gap threshold with a sloping configuration. were no signs of vertigo, tinnitus, or nasal disorders.

On examination, the patient was alert, with stable gait. Bilateral facial nerve palsy was observed involving mostly the lower branches of the facial nerve, as she had difficulty puffing up the cheeks and puckering the lips. House-Brackmann grade III was determined bilaterally. The other cranial nerves were intact. Both ears were full of granulation tissue and pus discharge. Therefore, the tympanic membranes could not be visualized. As for tuning-fork tests, the Weber test showed centralization while the Rinne test was negative bilaterally. Nasoendoscopy examination was unremarkable.

Blood investigations revealed normal white blood cell count, hemoglobin level, and platelet count. However, the erythrocyte sedimentation rate (ESR) was elevated to $48 \mathrm{~mm} / \mathrm{h}$, and C-reactive protein was elevated to $79 \mathrm{mg} / \mathrm{L}$. Her blood glucose was also elevated significantly, consistent with diabetes mellitus.

Pure-tone audiometry confirmed bilateral mild to severe conductive hearing loss with a sloping configuration (Figure 1). High-resolution computed tomography (HRCT) of the temporal petrous region demonstrated enhancing soft tissue at the skull base, just anterior to the clivus, extending to the bilateral periauricular regions and mastoid bone to obliterate the bilateral upper pharyngeal spaces (Figures 2, 3). The soft tissue mass also extended into the bilateral external auditory canals, causing bulging of tympanic membranes into the corresponding middle ears, and into the bilateral foramen lacerum with erosion of the adjacent petrous apex. Bony erosion was also seen in the bilateral inferior mastoid bones and external auditory canals. Both middle ears were spared from the lesions. Cultures of both ears exhibited growth of Pseudomonas aeruginosa. The technetium-99 bone scan showed uptake in the bilateral temporal regions (Figure 4).

She was hospitalized and subjected to aggressive treatment for MOE. Aural toileting was performed daily, and the ears were packed

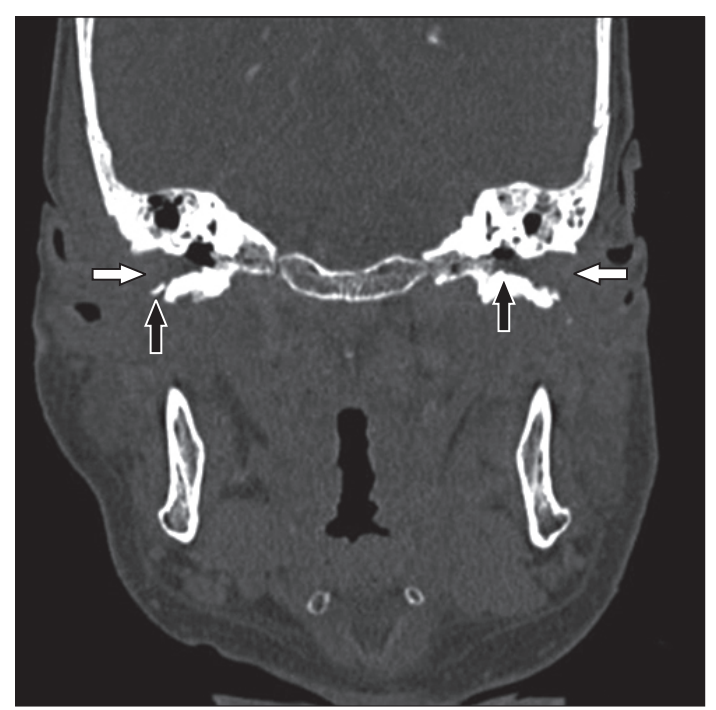

Figure 2. Coronal-view high-resolution computed tomography of the temporal bone shows a soft tissue mass (white arrows) in the bilateral ear canals with erosion (black arrows) of the underlying temporal bone. 


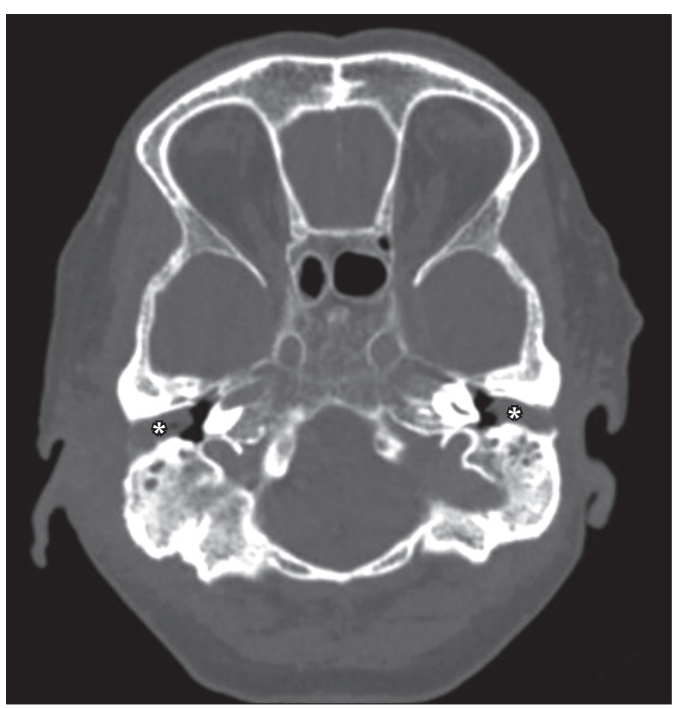

Figure 3. Axial-view high-resolution computed tomography of the temporal petrous shows the soft tissue mass * in bilateral ear canals with sparing of the middle ear.

with a ribbon gauze soaked with mupirocin and betamethasone creams. Intravenous ciprofloxacin $400 \mathrm{mg}$ was administered twice daily for 3 weeks, and strict control of newly diagnosed diabetes mellitus was achieved with insulin and oral hypoglycemic agents. Throughout the 3-week hospitalization period, the disease was monitored for improvements in ear pain, granulation tissue in the ear canal, ear discharge, facial nerve status, and blood parameters.

At the end of 3 weeks, the patient reported of no painful sensations, even without the use of analgesia. The granulation tissue become much smaller, and both the tympanic membranes were visible and intact. There was no ear discharge either. All the infective blood parameters reduced; particularly, the C-reactive protein level reduced from 79 to $3.4 \mathrm{mg} / \mathrm{L}$. She was discharged with a prescription of oral ciprofloxacin $500 \mathrm{mg}$ thrice daily for 3 months.

The patient remained well during the follow-up period at Otorhinolaryngology Clinic, Hospital Sultan Ismail. Pain, ear discharge, or granulation tissue seen in both ears did not recur. Facial nerve palsy improved to House-Brackmann grade II bilaterally. The patient was satisfied with her current condition.

The patient provided both verbal and written consent for publication of this case.

\section{DISCUSSION}

MOE is an aggressive and potentially life-threatening infection involving the external ear canal, temporal bone, and adjacent skull base. Immunocompromised individuals, particularly the elderly with diabetes mellitus are most commonly affected. ${ }^{1)}$ Although a lot of organisms have been cultured in this disease, the most common organism is Pseudomonas aeruginosa. ${ }^{2)}$

MOE is not common, and furthermore, only a few cases of bilateral

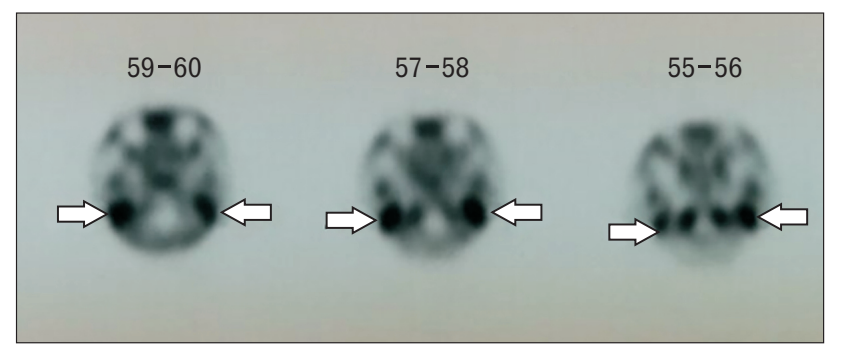

Figure 4. The technetium-99 bone scan shows uptake (white arrows) in bilateral temporal regions.

MOE have been reported. Giguere and Rouillard ${ }^{3)}$ reported a case of sequential MOE, which occurred in the subsequent 6 months. In the present case report, however, MOE interestingly occurred on both sides simultaneously, but the pain was more dominant in the left ear.

Why MOE predominantly presents unilaterally despite systemic risk factors, such as diabetes mellitus, is unknown. Clinical studies on this topic are limited, but Migirov et al. ${ }^{4)}$ postulated that it can be related to handedness. They reported that the right ear is commonly affected in right-handed people. Patients complained of ear itchiness, causing them to insert an object in the ear. However, more studies are warranted to confirm this theory.

The facial nerve is the most common cranial nerve involved, followed by the glossopharyngeal nerve, the vagus nerve and the accessory nerve. ${ }^{5)}$ This is due to its anatomic location in the temporal bone. ${ }^{2)}$

Facial nerve involvement indicates more progressive disease on computed tomography (CT), which verifies worsening of the disease by itself. ${ }^{6}$ However, it does not worsen the prognosis by itself. There is no difference in survival between those with facial nerve involvement and those without. ${ }^{6}$ Facial nerve palsy, coupled with bilateral ear involvements and other significant major HRCT findings, such as temporomandibular joint destruction, infratemporal fossa, or nasopharyngeal soft tissue involvement, do not show a poorer prognosis and tend to manifest as a prolonged, aggressive, and highly fatal disease. ${ }^{7)}$ The challenge in this patient was assessing the severity of the facial nerve paralysis. The face looked symmetrical because of the bilateral involvement of the facial nerve with the same severity. Sunny-Brook scoring, which evaluates the paralysis based on comparison with the normal side, was impractical, as the contralateral side was also affected. This is may have been easily overlooked at the local clinic or even by the emergency department personnel. Delay in detecting facial nerve palsy can lead to delay in treatment and predisposes the patient to developing complications.

For monitoring the disease progression, several modalities can be used. ESR is typically elevated in MOE, thus serving as a useful indicator for the response to therapy. ${ }^{2)}$ HRCT of the temporal bone is useful in diagnosing MOE but is not a proper tool for follow-up assessments. This is because the radiologic appearance of osteomyelitis is only evident when at least one-third of the bone mineral is lost, and conversely, bone remineralization continues long after the infection is cured. ${ }^{8)}$ 
The technetium-99 bone scan cannot be used to document resolution, as bone changes persist after disease resolution. ${ }^{9)}$ Ongoing infection is best monitored with ${ }^{67}$ Gallium scintigraphy, as ${ }^{67}$ Gallium is absorbed by macrophages and cells of the reticuloendothelial system, thus making it suitable for monitoring the response to treatment. ${ }^{10)}{ }^{67}$ Gallium scintigraphy combined with single-photon emission computed tomography/CT can help differentiate bone healing from active infection. ${ }^{9)}$ Further, if these scans reveal ongoing infection, antimicrobial therapy should be performed, and the scans can be repeated every 2-4 weeks until normalization. ${ }^{9)}$ We advocate that the patient should be followed-up for at least 6 months with a low threshold for imaging since MOE has a tendency to recur.

In conclusion, MOE is typically a unilateral disease but can present bilaterally in the ears. The presence of cranial nerve palsy needs to be identified early, as it indicates disease progression. Early diagnosis and prompt intervention can provide favorable outcome in an otherwise progressive and potentially fatal disease.

\section{CONFLICT OF INTEREST}

No potential conflict of interest relevant to this article was reported.

\section{ORCID}

Nur Adillah Lamry: https://orcid.org/0000-0001-7377-3897

Khairunnisak Misron: https://orcid.org/0000-0002-8656-1807

Tengku Mohamed Izam Tengku Kamalden: https://orcid.org/00000002-1388-6619

Azliana Aziz: https://orcid.org/0000-0002-6750-5797
Rosdan Salim: https://orcid.org/0000-0002-8724-9014

\section{REFERENCES}

1. Rubin Grandis J, Branstetter BF 4th, Yu VL. The changing face of malignant (necrotising) external otitis: clinical, radiological, and anatomic correlations. Lancet Infect Dis 2004;4:34-9.

2. Rubin J, Yu VL. Malignant external otitis: insights into pathogenesis, clinical manifestations, diagnosis, and therapy. Am J Med 1988;85:3918.

3. Giguere P, Rouillard G. Malignant bilateral external otitis in a 10-yearold girl. J Otolaryngol 1976;5:159-66.

4. Migirov L, Lipshitz N, Dagan E, Wolf M. Is laterality of malignant otitis externa related to handedness? Med Hypotheses 2013;81:142-3.

5. Corey JP, Levandowski RA, Panwalker AP. Prognostic implications of therapy for necrotizing external otitis. Am J Otol 1985;6:353-8.

6. Soudry E, Joshua BZ, Sulkes J, Nageris BI. Characteristics and prognosis of malignant external otitis with facial paralysis. Arch Otolaryngol Head Neck Surg 2007;133:1002-4.

7. Soudry E, Hamzany Y, Preis M, Joshua B, Hadar T, Nageris BI. Malignant external otitis: analysis of severe cases. Otolaryngol Head Neck Surg 2011;144:758-62.

8. Gold S, Som PM, Lucente FE, Lawson W, Mendelson M, Parisier SC. Radiographic findings in progressive necrotizing "malignant" external otitis. Laryngoscope 1984;94:363-6.

9. Courson AM, Vikram HR, Barrs DM. What are the criteria for terminating treatment for necrotizing (malignant) otitis externa? Laryngoscope 2014;124:361-2.

10. Garty I, Rosen G, Holdstein Y. The radionuclide diagnosis, evaluation and follow-up of malignant external otitis (MEO): the value of immediate blood pool scanning. J Laryngol Otol 1985;99:109-15. 